NOTICE: this is the author's version of a work that was accepted for publication in Trends in Food Science and Technology. Changes resulting from the publishing process, such as peer review, editing, corrections, structural formatting, and other quality control mechanisms may not be reflected in this document. Changes may have been made to this work since it was submitted for publication. A definitive version was subsequently published in Trends in Food Science and Technology, Vol. 38 (2014). DOI: 10.1016/j.tifs.2014.04.005 


\section{The emerging application of ultrasound in lactose crystallisation}

2 Authors: Mohammad H. Zamanipoor ${ }^{*}$, Ricardo L. Mancera

3 School of Biomedical Sciences, CHIRI Biosciences, Curtin University, GPO Box U1987,

4 Perth, WA 6845, Australia

$5 \quad$ *orrespondence: Mohammad H. Zamanipoor, School of Biomedical Sciences, Curtin

6 University, GPO Box U1987, Perth, WA 6845, Australia

7 e-mail: m.zamanipoor@gmail.com

8 Abstract: Ultrasonic processing is the industrial application of sound waves with a frequency

9 above the upper limit of human hearing. Interest has arisen recently in the effects of

10 ultrasound on the crystallisation of lactose as an innovative technology to improve its

11 recovery and the control over its crystal properties. This not only will increase the financial

12 profit for lactose manufacturers and improve the quality of lactose for specific applications,

13 but will also improve the quality of end products manufactured with lactose as an ingredient.

14 Short title: Ultrasonic crystallisation of lactose

15 Keywords: Lactose, crystallisation, ultrasound, recovery, particle engineering

16 Abbreviations: 7-ACDA: 7-amino-3-desacetoxy cephalosporanic acid; CSD: crystal size

17 distribution; DPI: dry powder inhaler; MSZW: metastable zone width 


\section{Introduction}

The first reports suggesting the ability of ultrasound to induce physical and chemical changes in materials were published in the late 1920s (Leonelli \& Mason, 2010; Richards, 1929; Richards \& Loomis, 1927; Wood \& Loomis, 1927). However, the use of ultrasound started to grow only after the 1970s due to the more general availability of commercial ultrasonic equipment (Leonelli \& Mason, 2010). The initial industrial applications of power ultrasound were in cleaning and plastic welding, which still continue to be the most popular applications (Mason, 2003). Due to the rapid growth of the technology in recent years, ultrasound has become a viable alternative option for some conventional food processing methods, such as emulsification, homogenisation and extraction (Ashokkumar et al., 2008; Patist \& Bates, 2008). Ultrasound has also been shown to improve other traditional processes such as filtration, extraction and crystallisation (Patist \& Bates, 2008).

Ultrasonic processing is the application of sound waves in the frequency range over $20 \mathrm{kHz}$, which is above human hearing (Leonelli \& Mason, 2010; Patel \& Murthy, 2009). Ultrasound has been categorised into 'high-intensity or power ultrasound' (20 - $100 \mathrm{kHz}$ ) (Suzuki, Lee, Padilla, \& Martini, 2010), which has usages in food processing, 'high-frequency ultrasound' $(100 \mathrm{kHz}-1 \mathrm{MHz})$ and 'diagnostic ultrasound' (1 - $10 \mathrm{MHz})$, which has medical applications (Patist \& Bates, 2008). However, the majority of food processing studies have been limited to the range of $20-40 \mathrm{kHz}$ (Mason, 1998), due to the higher physical effects (cavitation) and insignificant chemical effects (radical production) at lower frequencies (Ashokkumar et al., 2010; Hem, 1967).

Most studies on ultrasonic crystallisation have focused on its use in the manufacture of fine chemicals and pharmaceuticals, in attempts to increase their compliance with strict standards (Ruecroft, Hipkiss, Ly, Maxted, \& Cains, 2005). Ultrasonic crystallisation is an underresearched area in food technology (Deora, Misra, Deswal, Mishra, Cullen, \& Tiwari, 2013), with only a small number of reports on its use in the crystallisation of food materials such as milk fat (Suzuki et al., 2010), sunflower oil (Arends, Blindt, Janssen, \& Patrick, 2003), ice (Chow, Blindt, Chivers, \& Povey, 2003) and lactose (Zamanipoor, Dincer, Zisu, \& Jayasena, 2013). 
Interest has arisen in the last few years in the study of the effects of ultrasound on the crystallisation of lactose, particularly its potential ability to induce desirable crystal properties and to improve lactose recovery (Bund \& Pandit, 2007b; Bund \& Pandit, 2007c; Dhumal, Biradar, Paradkar, \& York, 2008; Kougoulos, Marziano, \& Miller, 2010; Patel \& Murthy, 2009; Patel \& Murthy, 2010; Patel \& Murthy, 2011a; Patel \& Murthy, 2011b; Zamanipoor et al., 2013). This review paper aims to critically discuss this emerging technique to provide an overall perspective of the benefits of the application of ultrasound in lactose crystallisation.

\section{The process of crystallisation}

60

61

62

The process of crystallisation from a solution has three distinct phases: formation of supersaturation (due to the difference between the solute concentration and solubility), nucleation (appearance of crystals) and crystal growth (Brito \& Giulietti, 2007; Bund \& Pandit, 2007a).

\subsection{Formation of supersaturation}

At any given temperature, a maximum quantity of solute can be dissolved in a solvent (Brito \& Giulietti, 2007). When a solution is saturated with a solute, it is considered to be in a thermodynamic equilibrium. However, an increase in concentration above the saturation (solubility) point disrupts the equilibrium, forming supersaturation and under these conditions crystallisation may occur (Deora et al., 2013).

In a plot of the temperature versus solute concentration, the region between the solubility and super-solubility curves is called the metastable zone width (MSZW), as shown for lactose in Fig. 1. In this region, despite the presence of supersaturation, crystallisation does not occur spontaneously (Shi, Hartel, \& Liang, 1989; Wong, Bund, Connelly, \& Hartel, 2011b). The time elapsed between the formation of supersaturation and the spontaneous appearance of crystals is referred to as 'induction time'. The induction time is a function of supersaturation and decreases with an increase in supersaturation (Luque de Castro \& Priego-Capote, 2007; Patel \& Murthy, 2009). 
Long induction time and wide MSZW are the factors responsible for the slow crystallisation of lactose, which necessitates very high supersaturation to induce nucleation (Dhumal et al., 2008; Patel \& Murthy, 2009; Patel \& Murthy, 2012; Raghavan, Ristic, Sheen, \& Sherwood, 2001). Seed addition and the use of anti-solvents have been used to try to reduce the induction time and accelerate the crystallisation of lactose (Dhumal et al., 2008).

\subsection{Nucleation and growth}

Nucleation involves the initial formation of crystals in a supersaturated solution (Brito \& Giulietti, 2007). It is an activated process in which the transition state is associated with the binding of molecules through intermolecular forces, such as hydrogen bonds, $\pi-\pi$ and van der Waals interactions (McLeod, 2007). As shown in Fig. 2, nucleation can be induced in two different pathways: (1) spontaneous nucleation, which can only happen at very high levels of supersaturation in the labile zone (primary, homogeneous nucleation), and (2) nucleation induced by a solid interphase (such as a container wall or a pre-existing particle, in which case it is called primary, heterogeneous nucleation; or a crystal of the solute, in which case it is called secondary nucleation). Secondary nucleation occurs because the crystals of the solute can either act as templates for the formation of new nuclei or break up to form further new nuclei (Luque de Castro \& Priego-Capote, 2007).

Once the nuclei are formed, they grow into large crystals in a growth process (RodríguezHornedo \& Murphy, 1999). The following mechanisms are generally assumed to be involved in crystal growth: bulk diffusion, surface diffusion and integration of growth units into the crystal surface. The growth rate is determined by the growth-limiting step, which is the slowest of the above mechanisms (Farhadi \& Babaheidary, 2002; McLeod, 2007). For $\alpha$ lactose, surface integration is reported to be the rate-limiting step (Thurlby, 1976).

Supersaturation is the driving force for nucleation and growth (Visser, 1982). Spontaneous primary nucleation cannot occur inside the MSZW since the energy available in the supersaturation in this region is not adequate to induce nuclei formation (Shi et al., 1989). However, it is possible to induce nucleation in the upper region of the MSZW (the area between the forced crystallisation and supersolubility curves in Fig. 1) using seeding or forced nucleation (Shi et al., 1989; Wong et al., 2011b). 
Both nucleation and the growth of crystals are system specific and highly dependent on supersaturation. Hence, increased control over nucleation and the growth of crystals and consequently on crystal properties can be achieved by controlling the supersaturation. Although both nucleation and the crystal growth occur rapidly at higher supersaturations in the labile zone, it is generally desirable not to increase the supersaturation to such high levels. Operating in this region, while promoting crystal growth, causes uncontrollable nucleation leading to a low mean crystal size and a wide crystal size distribution (CSD) (Patel \& Murthy, 2009; Patil, Gore, \& Pandit, 2008; Paul, Tung, \& Midler, 2005). A wide CSD is undesirable due to the resulting difficulties in processing such as in centrifugation, filtration and washing, leading to reduced recovery and poor final product quality (Shi, Liang, \& Hartel, 2006).

In order to achieve large particles with minimal CSD it is necessary to maximise growth and minimise secondary nucleation (Wong et al., 2011b). A growth-dominated process also has other advantages, such as the formation of crystals with lower surface area (easier to wash and dry, with lower entrapment of mother liquor), higher bulk density (easier to pack) and reduced formation of agglomerates (Paul et al., 2005). Paul et al. (2005) suggested that, in order for crystal growth to dominate over nucleation, it is necessary to control and limit the supersaturation. Since nucleation is more energy-demanding than growth (RodríguezHornedo \& Murphy, 1999), it can be assumed that under limited supersaturation, i.e. within the MSZW, crystal growth will dominate. Consequently, in order to achieve large crystals with minimal CSD, it has been proposed to operate the process of crystallisation inside the MSZW and use seeding to induce nucleation (Wong, Bund, Connelly, \& Hartel, 2011a; Wong et al., 2011b). On the other hand, in order to achieve reliable crystallisation performance, it has been suggested that operation should be carried out in the labile zone away from the MSZW (O’Grady, Barrett, Casey, \& Glennon, 2007). Consequently, it can be inferred that in conventional crystallisation, the conditions that enable control over the crystallisation process and crystal properties (i.e. operating at low supersaturation levels inside the MSZW) cannot offer the maximum process efficiency and performance. 


\section{Ultrasonic crystallisation}

136 Ultrasonic crystallisation is the use of power ultrasound which is mainly applied during the nucleation phase to control the crystallisation process (Bund \& Pandit, 2007c; Deora et al., 2013).

Ultrasound is transmitted as a series of compression and rarefaction cycles. Rarefaction cycles increase molecular distances by overcoming the attractive forces between molecules in the liquid and form cavities which continue to grow in size to create cavitation bubbles (Leonelli \& Mason, 2010). The bubbles then disperse throughout the liquid in the form of filament patterns due to the interaction forces with the sound field and other bubbles (Lauterborn, Kurz, Geisler, Schanz, \& Lindau, 2007). Many thousands of cavitation bubbles are generated, some of which are rather stable (stable cavitation), but most others grow into an unstable size at which point they collapse violently (transient cavitation), releasing a powerful shockwave (Deora et al., 2013; Leonelli \& Mason, 2010) (Fig. 3).

Three mechanisms have been proposed in the literature which supposedly promote nucleation in a crystallisation process as affected by ultrasound: (1) the shockwave released from cavitation bubbles promotes mass transfer and molecular collisions leading to the formation of primary nuclei (Cains, Martin, \& Price, 1998; Dhumal et al., 2008; Guo, Jones, \& Li, 2006b; Guo, Zhang, Li, Wang, \& Kougoulos, 2005; Luque de Castro \& Priego-Capote, 2007; Patel \& Murthy, 2009), (2) the aeration caused by the formation and movement of cavitation bubbles promotes mass transfer ( $\mathrm{Li}, \mathrm{Li}, \mathrm{Guo}, \& \mathrm{Liu}, 2006)$, and (3) the evaporation from the internal surface of the bubbles results in localised cooling, leading to the development of very high internal supersaturation and nucleation, which enables the bubbles to act as nucleation centres (Hem, 1967). Although these mechanisms generally agree that ultrasound promotes nucleation, there is no consensus regarding the effect of ultrasound on crystal growth. Some reports have indicated that ultrasound promotes crystal growth (Li et al., 2006; Mason, 1998), while others have reported obtaining smaller crystals by sonication and/or highlighted the effect of ultrasound on the disruption of grown crystals (Chow et al., 2003; Hem, 1967; Patel \& Murthy, 2009; Patel \& Murthy, 2011a; Suzuki et al., 2010). 
Guo et al. (2005) and Li et al. (2006) studied the effect of ultrasound on the induction times of roxithromycin and 7-amino-3-desacetoxy cephalosporanic acid (7-ACDA), respectively, and reported that the induction time of the sonicated sample was significantly shorter than the control, especially at lower supersaturations. In other words, the induction time of the sonicated sample at lower concentrations was shorter than that for the non-sonicated sample at higher concentrations. They concluded that sonication can narrow the MSZW and induce nucleation at lower supersaturations than conventional mixing. This reduction in induction time has been ascribed to an acceleration in diffusion induced by the ultrasonic power, suggesting a diffusion-controlled mechanism (Guo et al., 2005).

172 Guo et al. (2005) also reported a reduction in the crystal size of roxithromycin induced by 173 ultrasound and correlated it to the disrupting effect of shockwaves and abrasion between crystals, leading to the formation of smaller crystals. On the other hand, Li et al. (2006) reported obtaining larger 7-ACDA crystals using ultrasound and correlated it to the rapid growth and improved mass transfer induced by sonication.

Dalas (2001) reported no change in the typical rhombohedral morphology of calcite crystals and the spherical morphology of vaterite crystals as affected by ultrasound, and concluded that ultrasound did not result in the preferential growth of a certain crystal face. However, Guo et al. (2005) observed that ultrasound induced a change in the crystal shape of roxithromycin from hexagonal to rhombus, and Amara, Ratsimba, Wilhelm and Delmas (2001) noticed a change in the crystal shape of potash alum from octahedral to decahedral. They correlated the change in the typical crystal shape mainly to the fact that ultrasound can preferentially affect the growth rate of certain crystal faces.

Table 1 summarises the different reported effects of ultrasound on the crystallisation of a variety of chemical compounds. It is apparent that contrasting effects have been reported for different chemical compounds, and sometimes for the same chemical compound but under different experimental conditions. These contrasting findings highlight that the effects of ultrasound on a crystallisation system depend on the nature of the system. Hence, it is of the utmost importance that the behaviour of each crystallisation system as affected by ultrasound be investigated individually. Adjusting the ultrasonic variables to achieve desirable crystallisation effects will also depend on the findings of such investigations for each system. 


\section{Lactose and its crystallisation}

194

195

196

197

198

199

200

201

202

203

204

205

206

207

208

209

210

211

212

213

214

215

216

217

218

219

220

221

222

Lactose is the major carbohydrate in milk. It is a disaccharide composed of galactose and glucose linked by a $\beta(1 \rightarrow 4)$ glycosidic bond (Fox, 2009; Wong et al., 2011b). It is present in the milk of all mammals (with only a few exceptions) at an approximate concentration of 2 $10 \%$ by weight, and can be found in bovine milk at an average concentration of $4.8 \%$. Lactose has a level of sweetness about $20 \%$ that of sucrose. The crystals of $\alpha$-lactose monohydrate can be prepared by allowing a supersaturated aqueous solution to crystallise below $93.5^{\circ} \mathrm{C}$ (Gänzle, Haase, \& Jelen, 2008).

Lactose is derived from whey, which is the liquid that remains after milk has been curdled and strained, generally being a by-product in the process of cheese-making (Patel \& Murthy, $2011 \mathrm{~b})$. The annual worldwide production of whey is estimated to exceed 80 million tons per year, $40-50 \%$ of which is disposed of as sewage and the rest is used in human food and animal feed production (Cheryan, 2005). The direct disposal of whey into the environment can cause pollution, mainly due to its lactose content (approximately $5 \% \mathrm{w} / w$ ), which contributes to over $80 \%$ of the biological oxygen demand (BOD) of whey (Patel \& Murthy, 2009). Hence, the recovery of lactose from whey before discharging is necessary for the dairy industry to prevent potential risks to human and animal health. The manufacture of lactose also offers the advantage of improving the financial gain from whey utilisation by the production of a valuable ingredient with diverse food and pharmaceutical applications (Patel \& Murthy, 2009), such as in bakery goods and confectionery products (contributing to Maillard browning and enhancing flavour), meat products (filler and flavour enhancer), infant formulas (increasing lactose content to match human milk) and pharmaceutical products (filler and carrier).

The crystallisation of lactose is mainly carried out through evaporation to form supersaturation, followed by agitation in cooling crystallisers (Nickerson, 1970). In the industrial manufacture of lactose, whey usually undergoes purifications steps prior to being sent to the evaporators and crystallisers, since it contains impurities such as proteins, fats and minerals. The presence of impurities usually interferes with molecular movement and orientation, hence retarding or even inhibiting crystallisation. Proteins and salts contaminate lactose and reduce its purity, and increase the viscosity of concentrated whey, making the 
separation of crystallised lactose extremely difficult (Nickerson, 1970). Furthermore, the presence of mineral impurities in the final lactose product renders it unsuitable for most food and pharmaceutical applications, which require an ash content of $<0.2$ and $<0.1 \%$, respectively (Lifran et al., 2011). Consequently, purification steps such as demineralisation, heat coagulation, centrifugation, ultrafiltration and nanofiltration are initially performed to bring whey to the highest possible level of lactose purity (Lifran et al., 2011; Patel \& Murthy, 2012).

Two major difficulties are associated with the crystallisation of lactose: it is slow (Patel \& Murthy, 2010; Raghavan et al., 2001) and hardly controllable. The mixing generated by agitators in cooling crystallisers is not uniform, which results in random fluctuations and local zones of excessive supersaturation ( $\mathrm{Li}$, Wang, Bao, Guo, \& Zhang, 2003). In these regions, the nuclei and crystals cohere to each other and form agglomerates, which reduce crystallinity, leading to an increase in the susceptibility of crystals to break and the consequent formation of blunt edges. The agglomerates also trap traces of mother liquor which detrimentally affects the purity of the final product (Li et al., 2006). The random fluctuations in supersaturation also cause uneven growth in crystals (Dhumal et al., 2008), leading to a wide CSD. Moreover, surface irregularities, such as crevices and dislocations, may occur in crystals due to insufficient agitation, as some molecules may not have enough time to find the correct orientation for binding (Dhumal et al., 2008; Li et al., 2006). Another disadvantage of conventional crystallisation is the inefficient mixing which occurs mostly at the interfaces of the macroscopic layers in the solution (Li et al., 2006). This results in molecules in many regions of the solution having fewer opportunities to collide with each other and form nuclei, which leads to a long induction time and poor nucleation rate.

A number of studies have been undertaken in the last few years to address the abovementioned issues and gain control over crystal properties and improve lactose recovery by using ultrasound.

\section{The effect of ultrasound on nucleation rate of lactose}

The characterisation of the nucleation rate is of great importance in understanding and consequently achieving better control over the crystallisation process of lactose. Many crystal 
properties, such as crystal size and distribution as well as yield are highly influenced by the nucleation rate (Jiang \& ter Horst, 2010). The study of the nucleation rate of lactose is also important since lactose crystallises slowly and any process that can accelerate nucleation will result in huge cost savings for the lactose manufacturing industry by reducing the time required to manufacture each batch of lactose. The cost saving would be further improved if the nucleation of lactose can be induced at a low initial concentration, thus reducing evaporation costs (Patel \& Murthy, 2012).

Seeding and the use of anti-solvents have been used to try to accelerate the crystallisation of lactose (Dhumal et al., 2008). Seeding accelerates crystallisation by inducing secondary nucleation (forced crystallisation) and anti-solvents decrease solubility and hence increase supersaturation, which is the driving force for crystallisation (Visser, 1982). Although these methods improve the nucleation rate of lactose, both of them pose some major disadvantages.

The crystal habit is highly sensitive to the conditions and time of seed addition, which restricts the applicability and reproducibility of seeding (Dhumal et al., 2008; LouhiKultanen, Karjalainen, Rantanen, Huhtanen, \& Kallas, 2006). The quality of the final product is greatly influenced by the level of supersaturation present in the solution at the time of seed addition. For instance, if the seeds are introduced too early, when the solution is still undersaturated, a portion of the smaller seeds may dissolve. On the other hand, if seeding is performed too late, it usually exhibits no effect on the crystal attributes (Louhi-Kultanen et al., 2006). Another difficulty is that seeds are hard to disperse at the point of introduction into the solution and tend to agglomerate (Guo et al., 2005).

The use of anti-solvents poses its own problems. It has been reported to result in (1) wide batch-to-batch variability (O'Grady et al., 2007) because high supersaturation is formed rapidly and uncontrollably (Luque de Castro \& Priego-Capote, 2007), (2) formation of crystal agglomerates (Guo et al., 2005) and (3) fine and irregularly shaped crystals (O’Grady et al., 2007). This approach also requires expensive separation and purification steps to remove the anti-solvents from the product (Genck, 2010).

The use of ultrasound in crystallisation has been argued to shorten the MSZW and reduce the induction time. It can induce nucleation under low supersaturation conditions (inside the MSZW) where spontaneous nucleation cannot otherwise occur, therefore removing the need 
for seeding and reducing evaporation costs. It has been reported that under equal conditions, the effect of ultrasound on inducing nucleation is higher than increasing concentration (Luque de Castro \& Priego-Capote, 2007; Patel \& Murthy, 2009).

Ultrasound has been applied in lactose crystallisation primarily to accelerate nucleation. However, most studies on the ultrasonic crystallisation of lactose have not reported its effects on nucleation rate, presumably since it is very difficult to measure (Bund \& Pandit, 2007b; Dhumal et al., 2008; Kougoulos et al., 2010; Patel \& Murthy, 2009; Patel \& Murthy, 2010; Patel \& Murthy, 2011b). Due to the scarcity of the techniques to monitor nucleation at its molecular level, nucleation is often measured based on macroscopic properties $(\mathrm{Hu}, \mathrm{Hale}$, Yang, \& Wilson, 2001; Jiang \& ter Horst, 2010; McLeod, 2007; Rodríguez-Hornedo \& Murphy, 1999).

Patel and Murthy (2011a) studied the effects of ultrasound on the anti-solvent crystallisation of lactose using $n$-propanol. They estimated the total number of crystals per $\mathrm{mL}$ obtained at the end of sonication time using the average roundness, density and mean diameter of recovered lactose. They found that the number of crystals per $\mathrm{mL}$ increases and the size of crystals decreases with an increase in sonication time (2 $-8 \mathrm{~min})$, suggesting that continuous sonication promotes nucleation rather than crystal growth. However, the authors did not report the effect of the duration of sonication on nucleation rate, which is the rate of increase in crystal number over time.

Zamanipoor et al. (2013) systematically studied the effects of ultrasonic variables including amplitude and duration as well as lactose concentration on the nucleation rate of lactose in a pure aqueous solution. Absorbance measurements were used as an indirect method for estimating the nucleation rate. Nucleation rate was found to increase with an increase in lactose concentration and sonication amplitude (Fig. 4). It was postulated that higher supersaturation increases the driving force for crystallisation and higher amplitude promotes cavitation effects leading to an increase in nucleation rate. The nucleation rate was found to be insensitive to the duration of sonication. A 10.6-fold increase in nucleation rate in the sonicated sample compared to the control was also observed, highlighting the effect of ultrasound on the promotion of the nucleation rate. 


\section{The effect of ultrasound on growth rate of lactose}

312 In addition to nucleation rate, growth rate is an important factor underlying the crystal

313

314

315

316

317 attributes, such as shape and size and recovery of lactose (Bund \& Pandit, 2007c; Patil et al., 2008). Hence, it is important to determine the effects of ultrasound and sonication conditions on growth rate to increase control over the process of lactose crystallisation, thereby inducing desirable crystal properties and yield. Most of the studies related to the ultrasonic crystallisation of lactose have not reported the effect of ultrasound on growth rate (Bund \& Pandit, 2007b; Dhumal et al., 2008; Kougoulos et al., 2010; Patel \& Murthy, 2009; Patel \& Murthy, 2010; Patel \& Murthy, 2011b).

In a study of the effects of ultrasound on the anti-solvent crystallisation of lactose using $n$ propanol, Patel and Murthy (2011a) varied the sonication time (2 - $8 \mathrm{~min})$ and measured the growth rate indirectly. They observed a reduction in crystal growth rate when the duration of sonication was increased. They postulated that continuous sonication favours nucleation over growth, resulting in final crystals of smaller size and a consequent reduction in growth rate.

A more comprehensive study by Zamanipoor et al. (2013) reported the effects of ultrasonic variables including amplitude and duration as well as lactose concentration on the growth rate of lactose in a pure aqueous solution. Concentration was found to be the only factor that significantly affected the crystal growth rate of lactose, and the growth rate increased with an increase in concentration. An increase in concentration, at a certain temperature, increases the supersaturation which is the driving force for crystallisation (Visser, 1982), promoting both nucleation and growth rates.

\section{The effect of ultrasound on crystal size and CSD of lactose}

The crystal size and CSD of lactose are important properties from a manufacturing point of view. It is critical to be able to tailor the desirable crystal size for different applications of lactose with a minimal CSD. Obtaining a narrow CSD has been an important but difficult-toachieve target for lactose manufacturers since lactose crystallises uncontrollably. It is desirable to minimise the CSD because achieving uniformity and reducing variability within the manufactured product is advantageous from both processing and quality points of view. 
Secondary nucleation occurs during the growth of lactose crystals, which results in the formation of numerous small crystals and, consequently, in a wide CSD (Shi et al., 2006; Wong at al., 2011b). These small crystals make the final processing (centrifugation, filtration, washing, drying, etc.) difficult, resulting in reduced recovery and a final product of low quality (Wong, et al., 2011a; Wong et al., 2011b). Consequently, the reduction of the number of fine crystals and the narrowing of CSD will not only improve product quality but also will promote lactose recovery. In addition, it is necessary for lactose manufacturers to control crystal size and CSD to adhere to regulatory and marketing requirements. It is thus generally desirable to operate the crystallisation process of lactose under conditions that promote crystal growth and minimise secondary nucleation, leading to the formation of larger crystals with narrow CSD (Shi et al., 2006; Wong et al., 2011b).

Crystallisation is the main method to produce pharmaceutical products (Patel \& Murthy, $2011 \mathrm{~b}$ ). It is noteworthy to mention that the lactose crystals required for the manufacture of pharmaceutical products should be of small size. It is more desirable to use fine lactose crystals as fillers in tablets, since small crystal size allows better blending with other drug ingredients (Dhumal et al., 2008). Lactose is the most commonly used carrier in dry powder inhalers (DPIs) to deliver the drug to the lower airways in lungs (Kaialy, Ticehurst, \& Nokhodchi, 2012; Steckel, Markefka, teWierik, \& Kammelar, 2004). The popularity of lactose for DPI applications arises from a combination of appropriate characteristics: high stability, safety, low cost and good flow properties (Kaialy et al., 2012; Smyth \& Hickey, 2005). A reduction in lactose crystal size has been show to improve the aerosolisation of albuterol sulfate in Rotahaler ${ }^{\circledR}$ and budesonide in Spinhaler ${ }^{\circledR}$ (Steckel \& Müller, 1997; Zeng, Martin, Marriott, \& Pritchard, 2001). Optimum drug delivery to the lung airways occurs when particles are made in the size range of $2-6 \mu \mathrm{m}$ (Pritchard, 2001). The shape of particles also plays an important role because elongated crystals are dragged by the forces of the air stream for longer periods of time (Dhumal et al., 2008) and more easily release the drug particles during aerosolisation, due to potentially less drug-carrier interparticulate forces (Kaialy \& Nokhodchi, 2012; Nokhodchi, Kaialy, \& Ticehurst, 2011). Zeng et al. (2001) suggested that smaller crystal size, higher elongation ratio (needle-shape crystals) and smoother surface contribute to better flow properties, dispersibility in air and the particles remaining airborne, leading to deeper lung penetration and improved delivery of the drug. 
Consequently, it is necessary to tailor small crystal size and achieve high elongation ratios and surface smoothness if the lactose product is to be used in the pharmaceutical industry.

Micronisation has been used as a common, traditional method to reduce the crystal size of lactose required for DPIs, which is performed by fluid air jet milling (Shariare, de Matas, York, \& Shao, 2011; Zeng et al., 2001). However, this technique is time-consuming, highly energy-inefficient, and also results in the formation of highly charged particles with high surface roughness, which significantly reduce the flowability required for efficient drug delivery (Kougoulos et al., 2010; Leonelli \& Mason, 2010; Zeng et al., 2001). Most importantly, it increases the amorphous (glass) content of the carrier, leading to reduced flow properties and dispersibility due to hygroscopicity (Ward \& Schultz, 1995; Zeng et al., 2001).

Another approach which has been used for the manufacture of small crystals for DPIs is the use of anti-solvents. It is well-established that the use of anti-solvents induces very rapid formation of supersaturation, which accelerates the growth of crystal length and a reduction in thickness, leading to the formation of smaller and elongated lactose crystals (Bund \& Pandit, 2007c; Patel \& Murthy, 2009; Patel \& Murthy, 2011b; Zeng et al., 2001), which are suitable for pharmaceutical applications. While the use of anti-solvents favours the desirable crystal size and shape for pharmaceutical applications, as described earlier, it also causes a number of difficulties.

A number of authors have reported the effect of ultrasound on the size and CSD of lactose crystals in solutions containing anti-solvents, with contrasting findings. It has been argued that applying ultrasound causes better mixing and distribution of the anti-solvent throughout the solution, leading to more uniform and rapid nucleation (Bund \& Pandit, 2007c; Patel \& Murthy, 2009; Patel \& Murthy, 2011b). Bund and Pandit (2007c) studied the crystal size and CSD of lactose as affected by ultrasound in a solution containing ethanol as an anti-solvent, and reported that protein is the dominant factor influencing CSD and crystal habit and that an increase in protein content $(0.2-0.8 \% w / v)$ widens the CSD. Furthermore, they found that an increase in lactose concentration $(11.5-17.5 \% \mathrm{w} / \mathrm{v})$ reduces the crystal size and narrows the CSD of lactose. These findings are consistent with those of Patel and Murthy (2009), who reported a reduction in crystal size and CSD of lactose with an increase in lactose concentration $(12-16 \% w / v)$ in a sonicated solution containing acetone as an anti-solvent. 
400

401

402

403

404

405

406

407

408

409

410

411

412

413

414

415

416

417

418

419

420

421

422

423

424

425

426

427

428

When crystallisation occurs in an anti-solvent system, an increase in lactose concentration could work synergistically with the anti-solvent, resulting in a more rapid formation of supersaturation and nucleation, leading to smaller crystal size. Interestingly, another study on the effect of ultrasound on lactose crystallisation with ethanol as anti-solvent reported no effect of lactose concentration $(20-30 \% \mathrm{w} / \mathrm{w})$ and sonication power $(10-30 \mathrm{~W})$ on crystal size (Kougoulos et al., 2010). The contrasting findings that have been reported with antisolvent systems may be associated to the type of anti-solvent used and the experimental conditions under which the studies were carried out.

Patel and Murthy (2011a) studied the effect of the duration of sonication on crystal size and CSD of lactose in a solution containing $n$-propanol as anti-solvent, and observed a reduction in crystal size and CSD when the sonication time increased from 2 to $8 \mathrm{~min}$. Kougoulos et al. (2010) also observed a decrease in the crystal size of lactose obtained from a reconstituted lactose solution containing ethanol when the sonication duration increased from 10 to 120 min. The above authors argued that continuous sonication induces secondary nucleation by cavitation disturbances at the crystal surfaces and breaks the already grown crystals, thus reducing crystal size. A similar observation was made by Dhumal et al. (2008), who reported obtaining smaller size crystals when an aqueous lactose solution was sonicated for $5 \mathrm{~min}$ compared to when it was sonicated for $45 \mathrm{~s}$, followed by $5 \mathrm{~h}$ of growth in a stagnant glycerin solution $(20 \% \mathrm{w} / \mathrm{w})$. Consequently, it can be inferred that the continuous use of ultrasound causes smaller crystals to be formed, regardless of the nature of the system investigated. This suggests that it should be possible to tailor the desirable crystal size by adjusting the duration of sonication (amongst other factors, such as lactose concentration).

\section{The effect of ultrasound on shape of lactose crystals}

Patel and Murthy (2011b) studied the ultrasonic crystallisation of lactose in the presence of acetone as an anti-solvent and reported obtaining needle/rod shaped crystals by sonication vs. tomahawk shape for the control (mechanically agitated at 1,000 rpm) and commercial lactose samples. They associated this to the mixing effect of ultrasound, which allows uniform supersaturation to be reached quickly, resulting in rapid crystallisation. Rapid crystallisation accelerates the growth of the longest axis and a decrease in width, resulting in the formation 
of needle-shaped, elongated crystals. Bund and Pandit (2007c) and Patel and Murthy (2011a) also reported obtaining needle and/or rod crystal habits as affected by ultrasound in lactose solutions containing the anti-solvents ethanol and $n$-propanol, respectively.

Kougoulos et al. (2010) studied ultrasonic crystallisation in a reconstituted lactose solution using ethanol as an anti-solvent. The addition time of ethanol was found to be the most significant factor on crystal habit and rod and needle-shaped crystals were mainly obtained when ethanol was added to the solution rapidly (within $10 \mathrm{~min}$ from the start of sonication), whereas a transition to tomahawk-shaped crystals was observed when the addition rate was slowed down to $120 \mathrm{~min}$, as shown in Fig. 5. Formation of the needle and rod crystal shapes was associated to the rapid increase in supersaturation at the nucleation point in the presence of ultrasound, which favours the formation of elongated crystals. It can be inferred from the transition observed in Fig. 5 that reducing the effect of the anti-solvent (by adding it slowly) leads to the formation of tomahawk-shaped crystals, which are not suitable for DPI applications. However, these tomahawk-shaped crystals could be fit for other particular uses such as food applications.

\section{The effect of ultrasound on lactose recovery}

Studying the ultrasonic crystallisation of lactose in aqueous solution, Zamanipoor et al., 2013 reported a significant, 5.6-fold increase in lactose recovery as a result of sonication. This was consistent with Dhumal et al., 2008, who reported a significant positive effect of sonication on the recovery of lactose in aqueous system. The main mechanism by which ultrasound promotes the recovery of lactose is through its effects on mass transfer and nucleation rate, as explained previously.

Bund and Pandit (2007c) and Patel and Murthy (2009) studied the ultrasonic crystallisation of lactose in the presence of the anti-solvents ethanol and acetone, respectively. A strong positive dependence of lactose recovery on the concentration of lactose $(11.5-17.5 \% \mathrm{w} / \mathrm{v}$ and $12-16 \% w / v$, respectively) was reported. This was explained by the fact that, in the presence of cavitation, higher concentration causes higher supersaturation to be formed, resulting in a higher nucleation rate and subsequent higher lactose recovery. By contrast, Patel and Murthy (2010) studied the ultrasonic crystallisation of lactose from paneer whey after de-fatting and 
de-proteination (reducing the protein content to less than $0.1 \% \mathrm{w} / \mathrm{v}$ ) and reported no significant effect of lactose concentration $(5-15 \% \mathrm{w} / \mathrm{w})$ on lactose recovery in a system containing acetone as an anti-solvent. This could be attributed to the inhibitory effect of the remaining protein and whey impurities on the nucleation rate, rendering the driving force of higher supersaturation ineffective in inducing significant nucleation.

Bund and Pandit (2007c) and Patel and Murthy (2011a) also reported a significant negative effect of the protein content of lactose solutions on lactose recovery. A reduction in yield was observed with the increase in protein content $(0-0.8 \% \mathrm{w} / \mathrm{v}$ and $0.2-0.8 \% \mathrm{w} / \mathrm{v}$, respectively), which was associated with a reduction in nucleation rate, since proteins are known to be crystallisation inhibitors (Bund \& Pandit, 2007c; Patel \& Murthy, 2011a). However, even in the presence of protein, the yield in the sonicated sample was higher than the control (Bund \& Pandit, 2007c).

Patel and Murthy (2009) reported that the recovery of lactose from reconstituted lactose solutions improved with an increase in sonication time $(2-8 \mathrm{~min})$ in the presence of acetone. A sharp increase in recovery was observed when the sonication time was increased from 2 to $4 \mathrm{~min}$, but a subsequent increase from 4 to $8 \mathrm{~min}$ did not seem to have a large influence on the yield. Other reports have also indicated a similar increase in yield with an increase in sonication time, reaching a plateau with prolonged sonication (Bund \& Pandit, 2007c; Patel \& Murthy, 2010; Patel \& Murthy, 2011a). It was postulated that this could be due to a decrease in supersaturation level as nucleation depletes the supersaturation over prolonged sonication times (Patel \& Murthy, 2011a). As the solution becomes depleted from supersaturation, any longer sonication does not result in any further recovery.

\section{Industrial scale-up and future trends}

Research on the ultrasonic crystallisation of a number of chemical compounds has generally shown promising results in terms of yield and product quality for the manufacturing industry. However, the ultrasonic crystallisation of lactose is still in its infancy and further studies are needed to fully characterise its parameters to maximise its benefits. In particular, future research should aim to describe how lactose crystals respond to ultrasonic variables, as well as to explore pathways to engineer and tailor desirable lactose crystals suitable for specific 
applications, while maximising lactose recovery. Such laboratory-scale studies are essential prior to investigating the scale-up of ultrasonic crystallisation of lactose. A critical factor, among many others, is the duration of sonication required to achieve desirable results, since the type of system required for scale-up (batch or continuous) will primarily depend on the time period required for sonication.

There is a significant lack of knowledge in the area of ultrasonic crystallisation of lactose for food applications, which generally require larger crystals with tomahawk shapes and narrow CSD. Achieving the desirable lactose product quality and enhancing its recovery will not only save the lactose manufacturing industry million dollars per year, but will also improve the quality of the food products manufactured with lactose as an ingredient.

In addition to efforts aimed at implementing ultrasound in lactose crystallisation in the industry, more powerful techniques to monitor nucleation and crystal growth need to be developed to characterise how they respond to ultrasound. In addition, scale-up efforts will be hampered if appropriate large-scale, food-grade devices able to deliver enough ultrasonic power to lactose concentrates are not manufactured.

The penetration range of power ultrasound in a liquid is limited, and is estimated to be around $10 \mathrm{~cm}$ from the tip of a probe (Li et al., 2003; McCausland, Cains, \& Martin, 2001). Consequently, in order to sonicate the large volumes required for industrial manufacturing, some technological developments have been initiated. These approaches include (1) increasing the number of ultrasonic probes in a batch tank or pumping the liquid through a number of probes in a tube (Li et al., 2003; Patel \& Murthy, 2012), (2) positioning 'tips' or small-area ultrasonic delivery devices in batch tanks or flow-cells and (3) arranging opposite parallel transducers around a tube through which the liquid flows (Patel \& Murthy, 2012) (Fig. 6). The latter approach reduces the erosion of the equipment due to distributing the power between a large number of transducers and concentrating the ultrasonic intensity towards the centre of the cylinder rather than the vessel walls (Ashokkumar et al., 2010; Ruecroft et al., 2005). It also delivers ultrasonic energy as uniformly as possible throughout the liquid volume, avoiding the phenomenon of cavitational blocking (acoustic decoupling), which arises when high power is delivered to the liquid from a single delivery point (Ruecroft et al., 2005). 
517 In addition to the physical aspects of sonication such as penetration range and the problem of 518 erosion, the potential chemical problem of formation of free radicals should be taken into 519 consideration. The local, high temperatures generated at the time of bubble collapse can lead 520 to the formation of highly reactive radicals, such as $\mathrm{OH}^{*}$ and $\mathrm{H}^{\circ}$ radicals in aqueous solution

521 (Ashokkumar et al., 2010). Depending on the type of food and processing conditions, these

522 free radicals may either enhance or reduce the quality of the sonicated food material.

523 However, the formation of free radicals can be minimised by sonicating at low frequencies

$524(20 \mathrm{KHz})$ or using appropriate radical scavengers at higher frequencies (Ashokkumar et al., 525 2008).

526 The scale-up and adaptation of ultrasonic equipment for lactose manufacturing can be 527 challenging and requires careful consideration. Most importantly, the scale-up equipment 528 must be free from contamination and should not undergo any erosion induced by ultrasonic 529 cavitation. Eroded materials (e.g. titanium alloys) migrate to the crystal slurry and 530 contaminate it, rendering it inappropriate for food and pharmaceutical applications due to 531 their health hazards. There is consequently a need to investigate these contamination 532 processes and find ways to manufacture equipment that eliminates it. Furthermore, it is 533 necessary to study any detrimental chemical changes that ultrasound may induce in the 534 lactose crystal slurry and which could impact the sensory and/or hygienic properties of the 535 final lactose product.

\section{11. Conclusions}

537 Ultrasonic processing is an emerging technology which has been generally shown to improve the crystallisation process of a number of chemical compounds, mainly improving control over the crystal properties and recovery. However, the ultrasonic crystallisation of lactose has not been researched extensively, especially in relation to food applications. Contrasting

541 findings have been reported for the use of ultrasound for different chemical compounds, 542 which necessitates in-depth investigation of its application for any crystallisation medium, 543 including lactose. Two major difficulties are associated with the crystallisation of lactose: it is 544 slow and hardly controllable, and different attempts have been focused to address these issues. The use of ultrasound has been shown to improve the recovery of lactose, and 
546 adjusting the processing variables such as duration of sonication and lactose concentration

547 can be useful in tailoring the desired lactose crystals for pharmaceutical applications, which

548 require small, elongated lactose crystals. By building upon knowledge gained from

549 pharmaceutical systems, it would be possible to engineer desirable lactose crystals for food

550 applications. The industrial scale-up of ultrasonic technology for the manufacture of lactose

551 still requires extensive laboratory-scale and pilot-scale studies directed at exploring pathways

552 to tailor desirable lactose crystals suitable for each application, while maximising lactose

553 recovery. In addition, appropriate large-scale, food- and pharmaceutical-grade equipment

554 able to deliver enough ultrasonic power to lactose concentrates need to be designed and 555 manufactured.

556 Acknowledgements

557 MHZ gratefully acknowledges the Australian Government and Curtin University for funding 558 his Ph.D. research.

\section{References}

560 Amara, N., Ratsimba, B., Wilhelm, A.-M., \& Delmas, H. (2001). Crystallization of potash

561 alum: effect of power ultrasound. Ultrasonics Sonochemistry, 8, 265-270.

562 Arakelyan, V. S. (1987). Effect of ultrasound on crystal growth from melt and solution. Acta

563 Physica Hungarica, 61, 185-187.

564 Arends, B. J., Blindt, R. A., Janssen, J., \& Patrick, M. (2003). Crystallization process using 565 ultrasound. United States Patent. US 6,630,185 B2.

566 Ashokkumar, M., Bhaskaracharya, R., Kentish, S., Lee, J., Palmer, M., \& Zisu, B. (2010).

567 The ultrasonic processing of dairy products - An overview. Dairy Science \& Technology, $56890,147-168$.

569 Ashokkumar, M., Sunartio, D., Kentish, S., Mawson, R., Simons, L., Vilkhu, K., \& Versteeg, 570 C. K. (2008). Modification of food ingredients by ultrasound to improve functionality: A 
preliminary study on a model system. Innovative Food Science \& Emerging Technologies, $9(2), 155-60$.

Brito, A. B. N., \& Giulietti, M. (2007). Study of lactose crystallization in water-acetone solutions. Crystal Research \& Technology, 42, 583-588.

Bund, R. K., \& Pandit, A. B. (2007a). Rapid lactose recovery from buffalo whey by use of 'anti-solvent, ethanol'. Journal of Food Engineering, 82, 333-341.

Bund, R. K., \& Pandit, A. B. (2007b). Rapid lactose recovery from paneer whey using sonocrystallization: A process optimization. Chemical Engineering \& Processing, 46 (9), 846-850.

Bund, R. K., \& Pandit, A. B. (2007c). Sonocrystallization: Effect on lactose recovery and crystal habit. Ultrasonics Sonochemistry, 14(2), 143-152.

Cains, P. W., Martin, P. D., \& Price, C. J. (1998). The use of ultrasound in industrial chemical synthesis and crystallization. 1. Applications to synthetic chemistry. Organic Process Research \& Development, 2(1), 34-48.

Cheryan, M. (2005). Dairy Technology. http://faculty.fshn.illinois.edu/ mcheryan/dairy.htm (accessed March 28, 2013).

Chow, R., Blindt, R., Chivers, R., \& Povey, M. (2003). The sonocrystallisation of ice in sucrose solutions: primary and secondary nucleation. Ultrasonics, 41, 595-604.

Dalas, E. (2001). The effect of ultrasonic field on calcium carbonate scale formation. Journal of Crystal Growth, 222, 287-292.

Deora, N. S., Misra, N. N., Deswal, A., Mishra, H. N., Cullen, P. J., \& Tiwari, B. K. (2013). Ultrasound for improved crystallisation in food processing. Food Engineering Reviews, 5, 3644.

Dhumal, R. S., Biradar, S. V., Paradkar, A. R., \& York, P. (2008). Ultrasound assisted engineering of lactose crystals. Pharmaceutical Research, 25(12), 2835-2844. 

sonocrystallization: Salbutamol sulphate for pulmonary delivery. International Journal of Pharmaceutics, 368, 129-137.

599 Farhadi, F., \& Babaheidary, M. B. (2002). Mechanism and estimation of $\mathrm{Al}(\mathrm{OH})_{3}$ crystal 600 growth. Journal of Crystal Growth, 234, 721-730.

601 Fox, P. F. (2009). Lactose: Chemistry and properties. In P. L. H. McSweeney, \& P. F. Fox 602 (Eds.), Advanced dairy chemistry, Vol. 3 (pp. 1-15). New York: Springer Science+Business 603 Media, LLC.

604 Gänzle, M. G., Haase, G., \& Jelen, P. (2008). Lactose: Crystallization, hydrolysis and value605 added derivatives. International Dairy Journal, 18, 685-694.

606 Genck, W. (2010). Make the most of antisolvent crystallization. Retrieved February 19, 2013 607 from http://www.chemicalprocessing.com/articles/2010/210/.

608 Guo, Z., Jones, A. G., \& Li, N. (2006a). Interpretation of the ultrasonic effect on induction 609 time during $\mathrm{BaSO}_{4}$ homogeneous nucleation by a cluster coagulation model. Journal of 610 Colloid \& Interface Science, 297, 190-198.

611 Guo, Z., Jones, A. G., \& Li, N. (2006b). The effect of ultrasound on the homogeneous 612 nucleation of $\mathrm{BaSO}_{4}$ during reactive crystallization. Chemical Engineering Science, 61(5), $613 \quad 1617-1626$.

614 Guo, Z., Zhang, M., Li, H., Wang, J., \& Kougoulos, E. (2005). Effect of ultrasound on anti615 solvent crystallization process. Journal of Crystal Growth, 273(3-4), 555-563.

616 Hem, S. L. (1967). The effect of ultrasonic vibrations on crystallization processes.

617 Ultrasonics, 5(4), 202-207.

618 Hu, H., Hale, T., Yang, X., \& Wilson, L. J. (2001). A spectrophotometer-based method for 619 crystallization induction time period measurement. Journal of Crystal Growth, 232(1-4), 8662092.

621 Hunziker, O. F. (1926). Condensed milk and milk powder. La Grange, Illinois. 
622

623

624

625

626

627

628

629

630

631

632

633

634

635

636

637

638

639

640

641

642

643

644

645

646

647

648

Jiang, S., \& ter Horst, J. H. (2010). Crystal nucleation rates from probability distributions of induction times. Crystal Growth \& Design, 11(1), 256-261.

Kaialy, W., \& Nokhodchi, A. (2012). Antisolvent crystallisation is a potential technique to prepare engineered lactose with promising aerosolisation properties: Effect of saturation degree. International Journal of Pharmaceutics, 437, 57-69.

Kaialy, W., Ticehurst, M., \& Nokhodchi, A. (2012). Dry powder inhalers: Mechanistic evaluation of lactose formulations containing salbutamol sulphate. International Journal of Pharmaceutics, 423, 184-194.

Kougoulos, E., Marziano, I., \& Miller, P. R. (2010). Lactose particle engineering: Influence of ultrasound and anti-solvent on crystal habit and particle size. Journal of Crystal Growth, 312(23), 3509-3520.

Lauterborn, W., Kurz, T., Geisler, R., Schanz, D., \& Lindau, O. (2007). Acoustic cavitation, bubble dynamics and sonoluminescence. Ultrasonics Sonochemistry, 14, 484-491.

Leonelli, C., \& Mason, T. J. (2010). Microwave and ultrasonic processing: Now a realistic option for industry. Chemical Engineering \& Processing: Process Intensification, 49(9), 885900.

Li, H., Li, H., Guo, Z., \& Liu, Y. (2006). The application of power ultrasound to reaction crystallization. Ultrasonics Sonochemistry, 13(4), 359-363.

Li, H., Wang, J., Bao, Y., Guo, Z., \& Zhang, M. (2003). Rapid sonocrystallization in the salting-out process. Journal of Crystal Growth, 247, 192-198.

Lifran, E. V., Sleigh, R. W., Johnson, R. L., Steele, R. J., Hourigan, J. A., \& Dalziel, S. M. (2011). Method for purification of lactose. United States Patent. US 2011/0034685 A1.

Louhi-Kultanen, M., Karjalainen, M., Rantanen, J., Huhtanen, M., \& Kallas, J. (2006).

Crystallization of glycine with ultrasound. International Journal of Pharmaceutics, 320(1-2), 23-29.

Luque de Castro, M. D., \& Priego-Capote, F. (2007). Ultrasound-assisted crystallization. Ultrasonics Sonochemistry, 14(6), 717-724. 
Lyczko, N., Espitalier, F., Louisnard, O., \& Schwartzentruber, J. (2002). Effect of ultrasound on the induction time and the metastable zone widths of potassium sulphate. Chemical Engineering Journal, 86, 233-241.

Mason, T. J. (1998). Power ultrasound in food processing - the way forward. In M. J. W. Povey, \& T. J. Mason (Eds.), Ultrasound in food processing (pp.105-126). London: Blackie Academic \& Professional.

Mason, T. J. (2003). Sonochemistry and sonoprocessing: the link, the trends and (probably) the future. Ultrasonics Sonochemistry, 10, 175-179.

McCausland, L. J., Cains, P. W., \& Martin, P. D. (2001). Use the power of sonocrystallization for improved properties. Chemical Engineering Progress, 97, 56-61.

McLeod, J. (2007). Nucleation and growth of alpha lactose monohydrate. PhD Thesis, Massey University.

Nickerson, T. A. (1970). Lactose. In B. H. Webb, \& E. O. Whittier (Eds.), Byproducts from milk (pp. 356-380). Westport: AVI Publishing.

Nishida, I. (2004). Precipitation of calcium carbonate by ultrasonic irradiation. Ultrasonics Sonochemistry, 11, 423-428.

Nokhodchi, A., Kaialy, W., \& Ticehurst, M. D. (2011). The influence of using carriers with increasing elongation ratios on uniformity, adhesion and in vitro aerosolization performance of salbutamol sulphate from dry powder inhalers. In: CRS Annual Meeting, Maryland, USA.

O’Grady, D., Barrett, M., Casey, E., \& Glennon, B. (2007). The effect of mixing on the metastable zone width and nucleation kinetics in the anti-solvent crystallization of benzoic acid. Chemical Engineering Research \& Design, 85(A7), 945-952.

Park, M.-W., \& Yeo, S.-D. (2010). Antisolvent crystallization of roxithromycin and the effect of ultrasound. Separation Science \& Technology, 45, 1402-1410.

Patel, S. R., \& Murthy, Z. V. P. (2009). Ultrasound assisted crystallization for the recovery of lactose in an anti-solvent acetone. Crystal Research \& Technology, 44(8), 889-896. 
675 Patel, S. R., \& Murthy, Z. V. P. (2010). Optimization of process parameters by Taguchi 676 method in the recovery of lactose from whey using sonocrystallization. Crystal Research \& 677 Technology, 45(7), 747-752.

678 Patel, S. R., \& Murthy, Z. V. P. (2011a). Anti-solvent sonocrystallisation of lactose. 679 Chemical \& Process Engineering, 32(4), 379-389.

680 Patel, S. R., \& Murthy, Z. V. P. (2011b). Effect of process parameters on crystal size and 681 morphology of lactose in ultrasound-assisted crystallization. Crystal Research \& Technology, 682 $46(3), 243-248$.

683

684

685

686

687

688

689

690

691

692

693

694

695

696

697

698

699

700

701

Patel, S. R., \& Murthy, Z. V. P. (2012). Lactose recovery processes from whey: A comparative study based on sonocrystallization. Separation \& Purification Reviews, 41(4), 251-266.

Patil, M. N., Gore, G. M., \& Pandit, A. B. (2008). Ultrasonically controlled particle size distribution of explosives: A safe method. Ultrasonics Sonochemistry, 15, 177-187.

Patist, A., \& Bates, D. (2008). Ultrasonic innovations in the food industry: From the laboratory to commercial production. Innovative Food Science \& Emerging Technologies, 9, 147-154.

Paul, E. L., Tung, H.-H., \& Midler, M. (2005). Organic crystallization processes. Powder Technology, 150, 133-143.

Pritchard, J. N. (2001). The influence of lung deposition on clinical response. Journal of Aerosol Medicine, 14 Suppl. 1, S19-26.

Raghavan, S. L., Ristic, R. I., Sheen, D. B., \& Sherwood, J. N. (2001). The bulk crystallization of $\alpha$-lactose monohydrate from aqueous solution. Journal of Pharmaceutical Sciences, 90(7), 823-832.

Richards, W. T. (1929). The chemical effects of high frequency sound waves II. A study of emulsifying action. Journal of the American Chemical Society, 51, 1724-1729.

Richards, W. T., \& Loomis, A. L. (1927). The chemical effects of high frequency sound waves I. A preliminary survey. Journal of the American Chemical Society, 49, 3086-3100. 
Rodríguez-Hornedo, N., \& Murphy, D. (1999). Significance of controlling crystallization mechanisms and kinetics in pharmaceutical systems. Journal of Pharmaceutical Sciences, 88, $651-660$

Ruecroft, G., Hipkiss, D., Ly, T., Maxted, N., \& Cains, P. W. (2005). Sonocrystallization: The use of ultrasound for improved industrial crystallization. Organic Process Research \& Development, 9, 923-932.

Santos, H. M., Lodeiro, C., \& Capelo-Martínez, J.-L. (2009). The Power of Ultrasound. In J.L. Capelo-Martínez (Ed.), Ultrasound in chemistry: analytical applications (pp. 1-16).

\section{Weinheim: Wiley-VCH Verlag GmbH \& Co. KGaA.}

Shariare, M. H., de Matas, M., York, P., \& Shao, Q. (2011). The impact of material attributes and process parameters on the micronisation of lactose monohydrate. International Journal of Pharmaceutics, 408, 58-66.

Shi, Y., Hartel, R. W., \& Liang, B. (1989). Formation and growth phenomena of lactose nuclei under contact nucleation conditions. Journal of Dairy Science, 72, 2906-2915.

Shi, Y., Liang, B., \& Hartel, R. W. (2006). Crystal refining technologies by controlled crystallization. United States Patent. US 2006/0128953 A1.

Smyth, H. D. C., \& Hickey, A. J. (2005). Carriers in drug powder delivery. American Journal of Drug Delivery, 3, 117-132.

Steckel, H., Markefka, P., teWierik, H., \& Kammelar, R. (2004). Functionality testing of inhalation grade lactose. European Journal of Pharmaceutics \& Biopharmaceutics, 57, 495505.

Steckel, H., \& Müller, B. W. (1997). In vitro evaluation of dry powder inhalers II: influence of carrier particle size and concentration on in vitro deposition. International Journal of Pharmaceutics, 154, 31-37.

Suzuki, A. H., Lee, J., Padilla, S. G., \& Martini, S. (2010). Altering functional properties of fats using power ultrasound. Journal of Food Science, 75, E208-E214. 
Thurlby, J. A. (1976). Crystallization kinetics of alpha lactose. Journal of Food Science, 41, $38-42$.

Virone, C., Kramer, H. J. M., van Rosmalen, G. M., Stoop, A. H., \& Bakker, T. W. (2006). Primary nucleation induced by ultrasonic cavitation. Journal of Crystal Growth, 294, 9-15.

Visser, R. A. (1982). Supersaturation of $\alpha$-lactose in aqueous solutions in mutarotation equilibrium. Netherlands Milk \& Dairy Journal, 36, 89-101.

Vu, T. T. L., Hourigan, J. A., Sleigh, R. W., Ang, M. H., \& Tade, M. O. (2003). Metastable control of cooling crystallisation. European Symposium on Computer Aided Process Engineering, 13, 527-532.

Ward, G. H., \& Schultz, R. K. (1995). Process-induced crystallinity changes in albuterol sulfate and its effect on powder physical stability. Pharmaceutical Research, 12, 773-779.

Wong, S. Y., Bund, R. K., Connelly, R. K., \& Hartel, R. W. (2011a, May). A systematic approach to optimization of industrial lactose crystallization. Paper presented at the $11^{\text {th }}$ International Congress on Engineering and Food, Athens, Greece. Retrieved February 20, 2013 from http://www.icef1 1.org/content/papers/fpd/FPD591.pdf.

Wong, S. Y., Bund, R. K., Connelly, R. K., \& Hartel, R. W. (2011b). Determination of the dynamic metastable limit for $\alpha$-lactose monohydrate crystallization. International Dairy Journal, 21(11), 839-847.

Wood, E. W., \& Loomis, A. L. (1927). The physical and biological effects of high-frequency sound-waves of great intensity. Phylosophical Magazine, 4, 417-436.

Zamanipoor, M. H., Dincer, T. D., Zisu, B., \& Jayasena, V. (2013). Nucleation and growth rates of lactose as affected by ultrasound in aqueous solutions. Dairy Science \& Technology, $93,595-604$.

Zeng, X.-M., Martin, G. P., Marriott, C., \& Pritchard, J. (2001). Lactose as a carrier in dry powder formulations: The influence of surface characteristics on drug delivery. Journal of Pharmaceutical Sciences, 90(9), 1424-1434. 
756 Fig. 1. Supersolubility diagram for lactose (reproduced from Wong et al., 2011b, with kind 757 permission from Elsevier; original data sources: Hunziker (1926) and Vu et al. (2003)).

758 Fig. 2. Classification of nucleation processes (reproduced from Luque de Castro \& Priego759 Capote, 2007, with kind permission from Elsevier).

760 Fig. 3. Creation of (a) transient and (b) stable cavitation bubbles. C and R denote 761 compression and rarefaction, respectively (reproduced and modified from Santos et al., 2009, 762 with kind permissions from Wiley-VCH Verlag GmbH \& Co. KGaA).

763 Fig. 4. Model for lactose nucleation rate as affected by ultrasound amplitude and 764 concentration (reproduced from Zamanipoor et al., 2013, with kind permission from Springer 765 Science and Business Media).

766 Fig. 5. Scanning electron microscopy (SEM) images showing the effect of the rate of ethanol 767 addition on lactose crystal habit in ultrasonic crystallisation: (a) addition within 10 min, (b) 768 addition within $60 \mathrm{~min}$, and (c) addition within $120 \mathrm{~min}$ from the start of sonication. The 769 CSD in all cases seems large, presumably due to the addition of anti-solvent (reproduced 770 from Kougoulos et al., 2010, with kind permission from Elsevier).

771 Fig. 6. Prosonix Prosonitron ${ }^{\circledR}$ P750 large-scale ultrasonic flow cell composed of 42 bonded 772 transducers, capable of delivering 1,200 W of power at $20 \mathrm{kHz}$ frequency. Printed with kind 773 permission from Prosonix Ltd., Oxford, UK. 